\title{
A Low-complexity Resource Optimization Technique for High Throughput Satellite
}

\author{
Tedros Salih Abdu, Steven Kisseleff, Eva Lagunas and Symeon Chatzinotas \\ Interdisciplinary Centre for Security, Reliability and Trust (SnT), \\ University of Luxembourg, Luxembourg \\ Email: \{tedros-salih.abdu, steven.kisseleff, eva.lagunas, symeon.chatzinotas\}@uni.lu
}

\begin{abstract}
The high throughput satellites with flexible payloads are expected to provide a high data rate to satisfy the increasing traffic demand. Furthermore, the reconfiguration capability of flexible payloads opens the door to more advanced system optimization techniques and a better utilization of satellite resources. Consequently, we can obtain high demand satisfaction at the user side. For this, dynamically adaptive high-performance and low-complexity optimization algorithms are needed. In this paper, we propose a novel low-complexity resource optimization technique for geostationary (GEO) High Throughput Satellites. The proposed method minimizes the transmit power and the overall satellite bandwidth while satisfying the demand per beam. This optimization problem turns out to be non-convex. Hence, we convexify the problem using Dinkelbach method and Successive Convex Approximation (SCA). The simulation result shows that the proposed scheme provides better flexibility in resource allocation and requires less computational time compared to the state-of-art benchmark schemes.
\end{abstract}

Index Terms-Bandwidth optimization, Dinkelbach method, Demand satisfaction, Flexible payloads, Low-complexity, Power optimization, Successive convex approximation.

\section{INTRODUCTION}

The Satellite Communication (SatCom) system provides a broad service from traditional media broadcasting to the internet-based broadband application. Furthermore, the system is suitable for remote locations, maritime markets, and aeronautical markets [1]. Currently, high throughput satellites with flexible digital payloads are emerging to cope with the increasing demand, which comes along with emerging ubiquitous services and increasing number of users. Moreover, the reconfigurability of flexible payloads depending on satellite resource, channel condition, and demand provides means for a better resource management with high demand satisfaction. For this, dynamically adaptive high-performance and lowcomplexity optimization algorithms are needed [2], [3].

The optimization of the satellite resource involves the transmit power and system bandwidth allocation according to the demand and channel condition. Hence, the problem can be formulated in principle as a multi-objective optimization problem. In this context, several resource optimization techniques have

This work has been supported by the Luxembourg National Research Fund (FNR) under the project FlexSAT (C19/IS/13696663) and the AFR Grant INSAT - "Power and Bandwidth Allocation for INterference-Limited SATellite Communication Systems". been proposed in the literature to satisfy the per beam demand. Power and bandwidth allocation based on simulated annealing algorithm to match the per beam demand have been considered in [4]. Furthermore, a Genetic Algorithm (GA) to optimize the power and bandwidth according to the demand request is proposed in [5]. Similarly, a GA with a Particle Swarm algorithm for satellite resource optimization has been considered in [6]. In [7], [8] Machine learning (ML) approach is used to manage the resource of the satellites. The proposed ML techniques require extensive training data to train the neural network. Additionally, the accuracy of the results depends on the type of training data, activation function, and the number of hidden layers. Generally, the metaheuristic and ML methods mentioned above suffer from local optimality, which degrades the demand satisfaction. Hence, optimal or close-to-optimum solution can not be guaranteed [9].

Analytical-based joint power and carrier frequency optimization to match the per beam demand introduced in [10]. First, the carriers required per beam using the Rayleigh quotient method is obtained. Then, equal power is assigned for each carrier frequency. However, the power allocation per carrier frequency is considered to be uniform. In [11], carrier and transmit power are optimized jointly to satisfy the per beam demand while minimizing the overall satellite resources. However, the dimension of the decision variables that need to be optimized is large, which leads to a high computational complexity and time consumption. Similarly, to study the limits of resource allocation without precoding, joint power and carrier allocation have been considered in [12]. However, the optimization focuses on maximization capacity, which does not depend on the user's demand.

In this paper, we propose a low-complexity multi-objective optimization for high throughput GEO satellites to optimize power and bandwidth jointly according to demand with less computational time while using the minimum overall resource of the satellite. Unlike carrier optimization, we focus on bandwidth allocation, which is shared by all users. we apply Dinkelbach method and Successive Convex Approximation (SCA) to convexify the problem. The simulation result shows that the proposed scheme provides better flexibility in resource allocation and requires less computational time compared with benchmark schemes.

The remainder of the paper is organized as follows. Section 
II introduces the system model and problem formulation. Then, we provide the proposed solution in Section III. Finally, the numerical results and conclusion are provided in Section IV and Section V, respectively.

\section{System Model And Problem Formulation}

A downlink multibeam high throughput GEO satellite is considered in Ka-band with $N$ beams. The system bandwidth is $B_{\text {total }}$ and the available transmit power is $P_{\text {total }}$. We assume that the system serves a single user per beam. Hence, we will use the term beam and user interchangeably. Further, we assume all users share the same bandwidth chunk $B$. Also we denote $p_{i}$ the transmit power for the $i$ th user.

The channel $h_{i, j}$ from the $j$ th beam to the $i$ th user is given by

$$
h_{i, j}=\frac{\sqrt{G_{R} G_{i}[j]}}{4 \pi \frac{d_{i}}{\lambda}}
$$

where $\lambda$ is the wavelength of the carrier frequency, $G_{R}$ is the user antenna gain, $G_{i}[j]$ denotes the beam gain from the $j$ th beam towards the $i$ th user, and $d_{i}$ is the distance between the satellite and the $i$ th user. Then, the received signal-tointerference-plus-noise ratio (SINR) of the $i$ th user is given by

$$
\gamma_{i}=\frac{g_{i, i} p_{i}}{\sum_{j=1, j \neq i}^{N} g_{i, j} p_{j}+N_{0} B}
$$

where $g_{i, i}=\left|h_{i, i}\right|^{2}$ is channel power gain and $N_{0}$ is the noise spectral density. Therefore, the offered capacity for the $i$ th user is

$$
C_{i}=B \log _{2}\left(1+\gamma_{i}\right)
$$

Finally, given the $i$ th beam demand $D_{i}$, the normalized unmet system capacity is given by

$$
C_{\text {unmet }}=\sum_{i=1}^{N} \max \left(1-C_{i} / D_{i}, 0\right)
$$

The offered capacity $C_{i}, \forall_{i}$, provided by the system should match the beam demand $D_{i}$ in order to guarantee its satisfaction. For this, the system needs to determine $B$ and $p_{i}$ to obtain minimum $C_{\text {unmet }}$. Moreover, the system should prevent allocating resources to beams where the demand is already satisfied. Therefore, the system is required to satisfy the per beam demand while using a minimum $B$ and $p_{i}$. Hence, we formulate a problem to minimize $B, p_{i}$ and $C_{\text {unmet }}$ under the system constraints. Accordingly, the optimization problem is formulated as

$$
\underset{B, p_{i}, \forall_{i}}{\operatorname{minimize}} \quad B+\sum_{i=1}^{N} p_{i}+\sum_{i=1}^{N} \max \left(1-C_{i} / D_{i}, 0\right)
$$

subject to:

$$
\begin{aligned}
& L 1: \gamma_{i} \geq \gamma^{\text {min }}, \forall_{i}, \\
& L 2: \sum_{i=1}^{N} p_{i} \leq P_{\text {total }}, \\
& L 3: p_{i} \leq P_{\text {max }}, \forall_{i} \\
& L 4: B \leq B_{\text {total }} \\
& L 5: B \geq B_{c} \\
& L 6: p_{i} \geq 0, \forall_{i}
\end{aligned}
$$

The constraint $L 1$ guarantees the minimum SINR requirement by the system. $L 2$ states that the overall used power must not exceed the total system power. Similarly, L3 refers to the transmit power not exceeding the maximum power $P_{\max }$ per beam. $L 3$ constraint ensures that the allocated bandwidth must not exceed the total bandwidth $B_{\text {total }}$. Furthermore, the constraint $L 5$ guarantees the minimum bandwidth requirement by the system. Finally, $L 6$ is a non-negative power constraint.

\section{LOW-COMPLEXITY RESOURCE OPTIMIZATION}

The non-differentiability of unmet system capacity and the non-linearity of $\gamma_{i}$ make the problem (5) non-convex. To transform (5) into a convex problem, first, we equivalently replace the unmet system capacity by slack variable $t_{i}, \forall_{i}$ by introducing additional constraints $t_{i} \geq 1-C_{i} / D_{i}, \forall_{i}$ and $t_{i} \geq 0, \forall_{i}$. Then, the equivalent repersentation of (5) is

$$
\underset{B, t_{i}, p_{i}, \forall_{i}}{\operatorname{minimize}} \quad B+\sum_{i=1}^{N} p_{i}+\sum_{i=1}^{N} t_{i}
$$

subject to:

$$
\begin{aligned}
& L 1, L 2, L 3, L 4, L 5, L 6, \\
& L 7: 1-\frac{C_{i}}{D_{i}} \leq t_{i}, \forall_{i}, \\
& L 8: t_{i} \geq 0, \forall_{i},
\end{aligned}
$$

Second, to avoid the product terms in $C_{i}$, we replace $B$ with $\frac{1}{T}$, where $T$ denotes the symbol interval. Then, we re-write the constraint $L 7$ as follows

$$
\hat{L} 7: T-\frac{\log _{2}\left(1+\gamma_{i}\right)}{D_{i}} \leq t_{i} T, \forall_{i}
$$

Furthermore, we replace the product term $t_{i} T$ by $s_{i}$ i.e., $s_{i}=t_{i} T, \forall_{i}$. Moreover, we express $\gamma_{i}$ as a function of spectral power density and noise spectral density:

$$
\gamma_{i}=\frac{g_{i, i} \widetilde{p}_{i}}{\sum_{j=1, j \neq i}^{N} g_{i, j} \widetilde{p}_{j}+N_{0}},
$$

where $\widetilde{p}_{i}=T p_{i}$ is the power spectral density per beam. 
The problem (6) is reformulated with the above modification as follows

$$
\begin{array}{ll}
\underset{\substack{T, s_{i}, \widetilde{p}_{i}, \forall_{i} \\
\text { subject to: }}}{\operatorname{minimize}} \quad \frac{1+\sum_{i=1}^{N} \widetilde{p}_{i}+\sum_{i=1}^{N} s_{i}}{T} \\
& \hat{L} 1: \gamma_{i} \geq \gamma^{\text {min }}, \forall_{i}, \\
& \hat{L} 2: \sum_{i=1}^{N} \widetilde{p}_{i} \leq T P_{\text {total }}, \\
& \hat{L} 3: \widetilde{p}_{i} \leq T P_{\text {max }}, \forall_{i}, \\
& \hat{L} 4: 1 \leq T B_{\text {total }}, \\
& \hat{L} 5: 1 \geq T B_{c}, \\
& \hat{L} 6: \widetilde{p}_{i} \geq 0, \forall_{i}, \\
& \hat{L} 7: T-\frac{\log _{2}\left(1+\gamma_{i}\right)}{D_{i}} \leq s_{i}, \forall_{i}, \\
& \hat{L} 8: s_{i} \geq 0, \forall_{i},
\end{array}
$$

The objective function and $\gamma_{i}$ are fractional functions. Hence, problem (9) remains non-convex. To convexify the objective function, let $\beta$ be optimal value of the objective function, i.e., $\frac{1+\sum_{i=1}^{N} \widetilde{p}_{i}+\sum_{i=1}^{N} s_{i}}{T}=\beta$. Hence, minimizing of (9) is equivalent to

$$
\underset{T, s_{i}, \widetilde{p}_{i}, \forall_{i}}{\operatorname{minimize}} 1+\sum_{i=1}^{N} \widetilde{p}_{i}+\sum_{i=1}^{N} s_{i}-\beta T
$$

subject to:

$$
\hat{L} 1, \hat{L} 2, \hat{L} 3, \hat{L} 4, \hat{L} 5, \hat{L} 6, \hat{L} 7, \hat{L} 8 \text {, }
$$

The formulation (10) is known as Dinkelbach method [13]. However, we can not apply directly Dinkelbach method because of fractional function $\gamma_{i}$. For this, we need to transform of $\gamma_{i}$ into a convex approximation function.

To convexify $\gamma_{i}$, first we bounded it by a lower bound slack variable $\Gamma_{i}$ as follows

$$
\begin{aligned}
& \hat{L} 7.1: T-\frac{\log _{2}\left(1+\Gamma_{i}\right)}{D_{i}} \leq s_{i}, \forall_{i}, \\
& \hat{L} 7.2: \Gamma_{i} \leq \frac{g_{i, i} \widetilde{p}_{i}}{\sum_{j=1, j \neq i}^{N} g_{i, j} \widetilde{p}_{j}+N_{0}}, \forall_{i},
\end{aligned}
$$

The constraint $\hat{L} 7.1$ is convex. However, $\hat{L} 7.2$ is non-convex. Re-arranging $\hat{L} 7.2$ :

$$
\hat{L} 7.2: \sum_{j=1, j \neq i}^{N} g_{i, j} q_{j}^{2}+N_{0}-\frac{g_{i, i} q_{i}^{2}}{\Gamma_{i}} \leq 0, \forall_{i}
$$

where $q_{i}=\sqrt{\widetilde{p}_{i}}, \forall_{i}$. The function $\sum_{j=1, j \neq i}^{N} g_{i, j} q_{j}^{2}+N_{0}$ and $\frac{g_{i, i} q_{i}^{2}}{\Gamma_{i}}$ are convex functions [14]. Therefore, $\hat{L} 7.2$ is arranged in the form of Difference-of-Convex program function (DC). The DC program can be tackled using Successive Convex Approximation (SCA) algorithm (cf. [15]) by approximating the concave part of $\hat{L} 7.2$. Hence, the SCA of $\hat{L} 7.2$ using firstorder approximation is

$$
\begin{aligned}
\hat{L} 7.3: & \sum_{j=1, j \neq i}^{N} g_{i, j} q_{j}^{2}+N_{0} \\
& +\frac{g_{i, i}\left(q_{i}^{(v)}\right)^{2}}{\left(\Gamma_{i}^{(v)}\right)^{2}} \Gamma_{i}-2 \frac{g_{i, i} q_{i}^{(v)} q_{i}}{\Gamma_{i}^{(v)}} \leq 0, \forall_{i},
\end{aligned}
$$

where $\Gamma_{i}^{(v)}$ and $q_{i}^{(v)}$ is the previous value of $\Gamma_{i}$ and $q_{i}$, respectively. Hence, the convexified optimization problem is written as

$$
\underset{T, \Gamma_{i}, s_{i}, q_{i}, \forall_{i}}{\operatorname{minimize}} 1+\sum_{i=1}^{N} q_{i}^{2}+\sum_{i=1}^{N} s_{i}-\beta T
$$

subject to:

$$
\begin{aligned}
& \hat{L} 1: \Gamma_{i} \geq \gamma^{\min }, \forall_{i}, \\
& \hat{L} 2: \sum_{i=1}^{N} q_{i}^{2} \leq T P_{\text {total }}, \\
& \hat{L} 3: q_{i}^{2} \leq T P_{\max }, \forall_{i}, \\
& \hat{L} 6: q_{i} \geq 0, \\
& \hat{L} 4, \hat{L} 5, \hat{L} 7.1, \hat{L} 7.3, \hat{L} 8,
\end{aligned}
$$

Finally, the normalized objective function of (14) is

$\underset{T, \Gamma_{i}, s_{i}, q_{i}, \forall_{i}}{\operatorname{minimize}} 1+\frac{B_{\text {total }}}{P_{\text {total }}} \sum_{i=1}^{N} q_{i}^{2}+B_{\text {total }} \sum_{i=1}^{N} s_{i}-\beta B_{\text {total }} T$

subject to:

$$
\hat{L} 1, \hat{L} 2, \hat{L} 3, \hat{L} 4, \hat{L} 5, \hat{L} 6, \hat{L} 7.1, \hat{L} 7.3, \hat{L} 8 \text {, }
$$

Problem (15) is convex and can be solved efficiently using convex optimization tools [16]. The iterative procedure to solve (15) is shown in Algorithm 1. First, we initialize $q_{i}^{(v)}, \Gamma_{i}$ and $\beta$ as shown in Algorithm 1.Then, for the next value of $v$, we solve (15). Subsequently, we update the old value of $q_{i}^{(v)}, \Gamma_{i}^{(v)}$ and $\beta$ by the new value $q_{i}$, $\Gamma_{i}$ and $\frac{\frac{1}{B_{\text {total }}}+\frac{1}{p_{\text {total }}} \sum_{i=1}^{N} q_{i}^{2}+\sum_{i=1}^{N} s_{i}}{T}$, respectively. Note that Algorithm 1 is designed to execute the Dinkelbatch method and SCA at the same time rather than considering Dinkelbatch method as main loop and SCA as inner loop. Hence, the computational time decreases. The algorithm updates the value $q_{i}^{(v)}, \Gamma_{i}^{(v)}$ and $\beta$ until the convergence criteria ${ }^{1}$ are met:

$$
f_{1}: \sum_{i}\left|\frac{g_{i, i}\left(q_{i}^{(v)}\right)^{2}}{\left(\Gamma_{i}^{(v)}\right)^{2}} \Gamma_{i}-\frac{g_{i, i} q_{i}^{(v)} q_{i}}{\Gamma_{i}^{(v)}}\right| \leq 10^{-4}
$$

and

$$
\begin{gathered}
f_{2}:\left|1+\frac{B_{\text {total }}}{P_{\text {total }}} \sum_{i=1}^{N} q_{i}^{2}+B_{\text {total }} \sum_{i=1}^{N} s_{i}-\beta B_{\text {total }} T\right| \\
\leq 10^{-4}
\end{gathered}
$$

${ }^{1}$ Note that in the convergence point we obtain $\Gamma_{i} \approx$
$\frac{g_{i, i}\left(q_{i}^{(v+1)}\right)^{2}}{\sum_{j=1, j \neq i}^{N} g_{i, j}\left(q_{j}^{(v+1)}\right)^{2}+N_{0}}$ and $\left(1+\frac{B_{\text {total }}}{P_{\text {total }}} \sum_{i=1}^{N} q_{i}^{2}+B_{\text {total }} \sum_{i=1}^{N} s_{i}-\right.$
$\left.\beta B_{\text {total }} T\right) \approx 0$. 


\section{Simulation Results}

In this section, we evaluate the performance of the proposed Low-Complexity Resource Optimization (LcRO) scheme. Table I shows the parameters required for the simulation. We obtained the results by averaging 100 Monte-Carlo iterations. Furthermore, we select a user per beam for each iteration using the Traffic simulator proposed in [17]. For comparison, we considered reference [4] and [11] as benchmark schemes. Additionally, for all simulation result, we consider heterogeneous demand $^{2}$ as shown in Fig. 1.

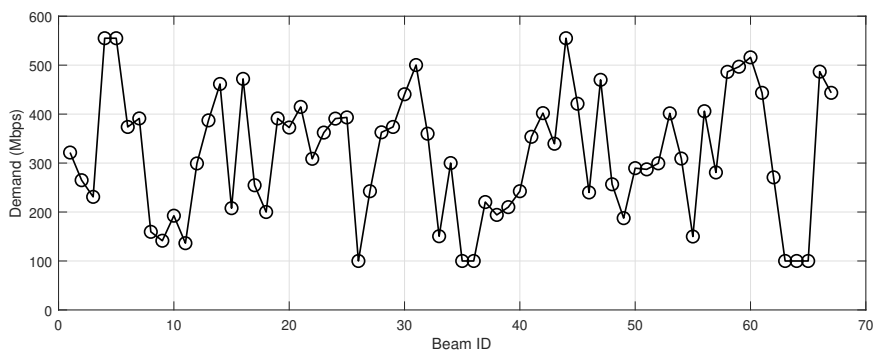

Fig. 1. The demand distribution of all beams.

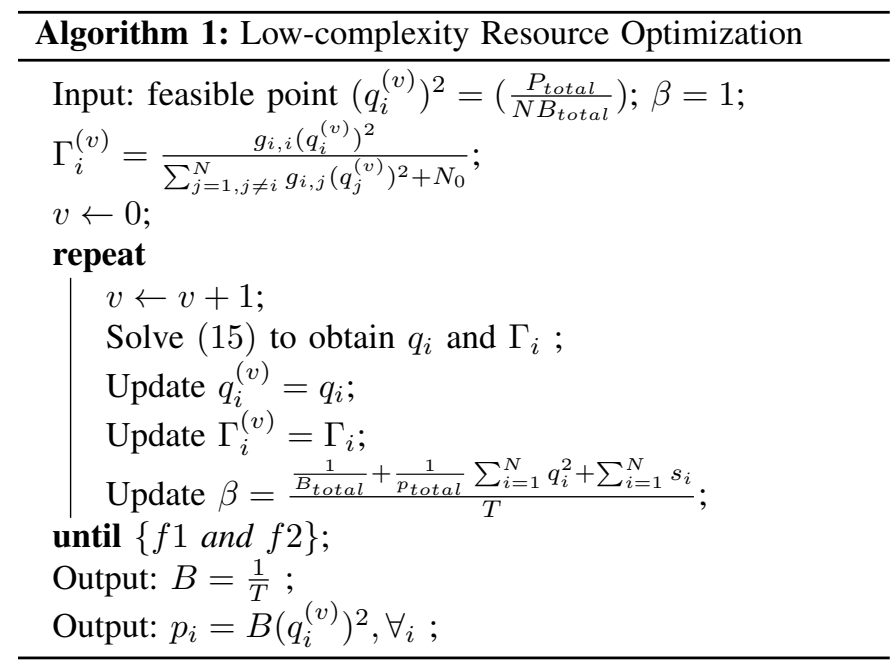

TABLE I

SYSTEM PARAMETERS

\begin{tabular}{|c|c|}
\hline Parameter & Value \\
\hline Satellite Orbit & $13^{\circ} \mathrm{E}$ \\
\hline Satellite Beam Pattern & Provided by ESA \\
\hline Number of beams $(N)$ & 67 \\
\hline System bandwidth $\left(B_{\text {tot }}\right)$ & $500 \mathrm{MHz}$ \\
\hline Minimum SINR $\left(\gamma^{\text {min }}\right)$ & $-2.2 \mathrm{~dB}$ \\
\hline Minimum bandwidth $\left(B_{C}\right)$ & $5 \mathrm{MHz}$ \\
\hline Noise power density $\left(N_{0}\right)$ & $-204 \mathrm{dBW} / \mathrm{Hz}$ \\
\hline Max. beam gain $\left(G_{i}[j]\right)$ & $51.8 \mathrm{dBi}$ \\
\hline User antenna gain $\left(G_{R}\right)$ & $39.8 \mathrm{dBi}$ \\
\hline Total available transmit power $\left(P_{\text {total }}\right)$ & $1000 \mathrm{~W}$ \\
\hline Maximum power per beam $\left(P_{\text {max }}\right)$ & $100 \mathrm{~W}$ \\
\hline
\end{tabular}

\footnotetext{
${ }^{2}$ The demand is carefully chosen to accommodate population-based demand, maritime demand and aeronautical demand [17].
}

The performance indicators used for the comparison are the average unmet system capacity, the Average utilized Bandwidth (AUB), the Average Power Consumption (APC), and the average satisfaction index [11]. Additionally, we define Total Resource Utilization (TRU) in percent as

$$
T R U=\frac{100}{2}\left(\frac{\mathrm{APC}}{P_{\text {total }}}+\frac{\mathrm{AUB}}{B_{\text {total }}}\right) \%
$$

Fig. 2 shows the Cumulative Distribution Function (CDF) of the computational time of the LcRO and the benchmark schemes. The LcRO has lower computational time compared with the benchmark schemes. For instance, the computing time of LcRO for $50 \%$ and $100 \%$ cases is $35 \mathrm{~s}$ and $55 \mathrm{~s}$, respectively, whereas, for [11], the computing time is $371 \mathrm{~s}$ and 700 s, respectively. Furthermore, the computing time of [4] for $50 \%$ and $100 \%$ cases is $122 \mathrm{~s}$ and 160 s, respectively. Hence, the computational complexity of the proposed techniques is less than the benchmarks schemes. This lower computational time is obtained because LcRO has less dimension of the optimization variables. Consequently, the computer needs a short time to execute each decision variable. In contrast, the benchmark schemes have high dimension of optimization variables. Hence, the computer needs more time to run to get a solution for each decision variable. For instance, the bandwidth $B$ of the LcRo is one variable, whereas the benchmark schemes assigns $\mathcal{K}$ carrier frequency to $N$ beams from $\mathcal{K} \times N$ optimization variables.

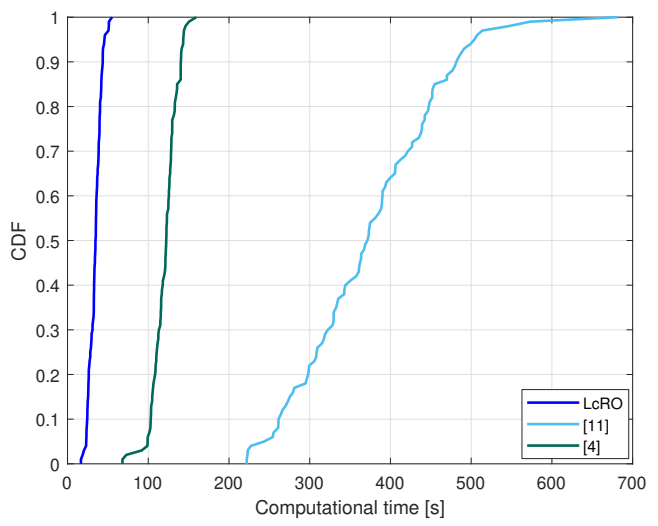

Fig. 2. Computational Time.

Fig. 3 shows the CDF of average unmet system capacity for all schemes. We observed that the proposed LcRO and [11] have zero unmet system capacity, whereas the unmet system capacity of [4] is higher than zero. For instance, at at $50 \%$ and $100 \%$ cases the unmet system capacity of [4] is $10 \mathrm{Mbps}$ and $65 \mathrm{Mbps}$, respectively. This high unmet system capacity results from the random allocation of resources to the beams. Hence, [4] is not optimal for demand satisfaction. In contrast, the LcRO and [11] use approximate convex algorithms that optimize the satellite's resource according to the demand. Hence, LcRO and [11] satisfy the per beam demand. Furthermore, the satisfaction index shown in Fig. 4 of LcRO and [11] for all beams is one, whereas the satisfaction index for the [4] scheme is lower for all beams. 


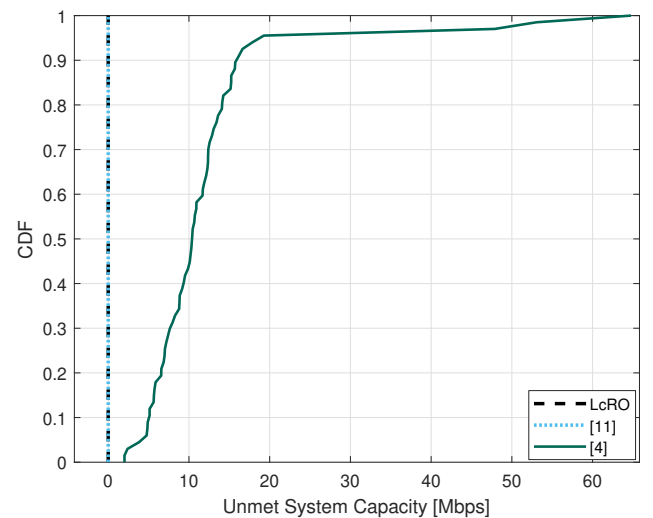

Fig. 3. The average unmet system capacity of LcRO and benchmark schemes.

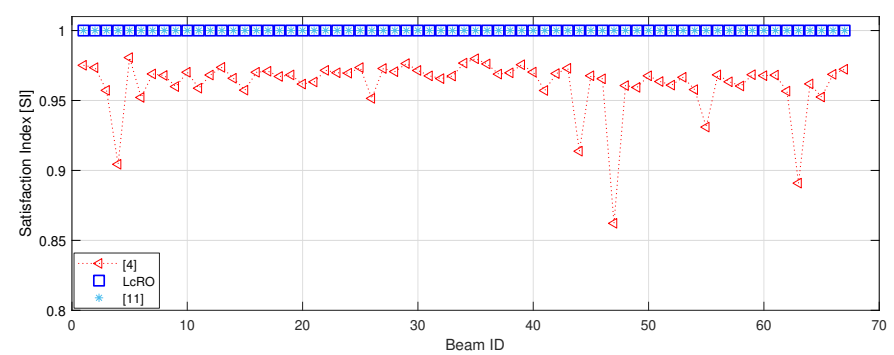

Fig. 4. The Average Satisfaction index of LcRO and benchmark schemes.

The resource utilization for LcRO, [11] and [4] is shown in Fig. 5. The total resource utilization for LcRO is $T R U=$ $\frac{100}{2}\left(\frac{249}{P_{t o t}}+\frac{391}{B_{t o t}}\right) \%=51.6 \%$. In contrast, for [11] the TRU is $T R U=\frac{100}{2}\left(\frac{146}{P_{t o t}}+\frac{498}{B_{t o t}}\right) \%=57.1 \%$ and for [4] the TRU is $T R U=\frac{100}{2}\left(\frac{527}{P_{t o t}}+\frac{500}{B_{t o t}}\right) \%=76.4 \%$. Hence, the proposed LcRO gives better resource utilization in addition to the substantial savings in computational complexity, as explained before.

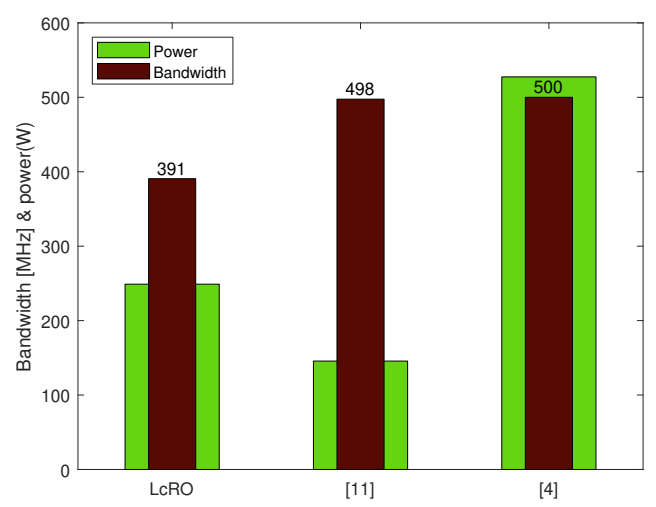

Fig. 5. The overall resource utilization of all schemes.

\section{CONCLUSiOnS}

In this paper, we proposed a novel low-complexity resource optimization technique for high throughput GEO Satellite to provide a high data rate with better resource utilization efficiency. The proposed method allocates power and bandwidth to satisfy the per beam demand while using the minimum overall satellite resource. Since the optimization problem is non-convex, we apply the Dinkelbach method and successive convex approximation to convexify the non-convex part of the problem. The simulation results show that the proposed scheme has better performance in flexible resource allocation and complexity than the benchmark schemes.

\section{REFERENCES}

[1] S. K. Sharma, S. Chatzinotas, and P.-D. Arapoglou, Satellite Communications in the $5 G$ Era. IET Digital Library, 2018.

[2] S. Kisseleff, E. Lagunas, T. S. Abdu, S. Chatzinotas, and B. Ottersten, "Radio resource management techniques for multibeam satellite systems," IEEE Communications Letters, pp. 1-1, 2020.

[3] O. Kodheli et al., "Satellite communications in the new space era: A survey and future challenges," IEEE Communications Surveys Tutorials, vol. 23, no. 1, pp. 70-109, 2021.

[4] G. Cocco, T. de Cola, M. Angelone, Z. Katona, and S. Erl, "Radio Resource Management Optimization of Flexible Satellite Payloads for DVB-S2 Systems," IEEE Transactions on Broadcasting, vol. 64, no. 2 pp. 266-280, Jun. 2018.

[5] A. Paris, I. Del Portillo, B. Cameron, and E. Crawley, "A Genetic Algorithm for Joint Power and Bandwidth Allocation in Multibeam Satellite Systems," in 2019 IEEE Aerospace Conference, March 2019, pp. 1-15.

[6] N. Pachler, J. J. G. Luis, M. Guerster, E. Crawley, and B. Cameron, "Allocating power and bandwidth in multibeam satellite systems using particle swarm optimization," in 2020 IEEE Aerospace Conference, 2020, pp. 1-11.

[7] P. V. R. Ferreira, R. Paffenroth, A. M. Wyglinski, T. M. Hackett, S. G. Bilén, R. C. Reinhart, and D. J. Mortensen, "Multiobjective reinforcement learning for cognitive satellite communications using deep neural network ensembles," IEEE Journal on Selected Areas in Communications, vol. 36, no. 5, pp. 1030-1041, 2018.

[8] F. G. Ortiz-Gomez, D. Tarchi, R. Martínez, A. Vanelli-Coralli, M. A. Salas-Natera, and S. Landeros-Ayala, "Convolutional neural networks for flexible payload management in vhts systems," IEEE Systems Journal, pp. 1-12, 2020.

[9] J. J. G. Luis, N. Pachler, M. Guerster, I. del Portillo, E. Crawley, and B. Cameron, "Artificial intelligence algorithms for power allocation in high throughput satellites: A comparison," in 2020 IEEE Aerospace Conference, 2020, pp. 1-15.

[10] J. Lei and M. A. Vázquez-Castro, "Multibeam satellite frequency/time Duality study and capacity optimization," Journal of Communications and Networks, vol. 13, no. 5, pp. 472-480, Oct 2011.

[11] T. S. Abdu, E. Lagunas, S. Kisseleff, and S. Chatzinotas, "Carrier and Power Assignment for Flexible Broadband GEO Satellite Communications System," in 2020 IEEE 31st Annual International Symposium on Personal, Indoor and Mobile Radio Communications, 2020, pp. 1-7.

[12] — - "Limits of Smart Radio Resource Assignment in GEO Satellite Communications," in IEEE Wireless Communications and Networking Conference (WCNC), 29th of March, accepted for presentation.

[13] W. Dinkelbach, "On nonlinear fractional programming," Management Science, vol. 13, no. 7, pp. 492-498, 1967. [Online]. Available: http://www.jstor.org/stable/2627691

[14] S. Boyd and L. Vandenberghe, Convex Optimization. Cambridge, UK: Cambridge Univ. Press, 2004.

[15] T. Wang and L. Vandendorpe, "Successive convex approximation based methods for dynamic spectrum management," in 2012 IEEE International Conference on Communications (ICC), 2012, pp. 4061-4065.

[16] M. Grant and S. Boyd, "CVX: Matlab software for disciplined convex programming, version 2.1," http://cvxr.com/cvx, Mar. 2014.

[17] H. Al-Hraishawi, E. Lagunas, and S. Chatzinotas, "Traffic simulator for multibeam satellite communication systems," in 10th Advanced Satellite Multimedia Systems Conference and the 16th Signal Processing for Space Communications Workshop (ASMS/SPSC), 2020, pp. 1-8. 